\title{
Mitochondria and Mitochondrion related organelles in Parasitic Protozoa-An Overview
}

\author{
A. Aiman, R. A. Shahardar and Z. A. Wani*
}

Division of Veterinary Parasitology, Faculty of Veterinary Sciences and Animal Husbandry, SKUAST- K, Shuhama (Alusteng) Srinagar, Kashmir- 190006

* Corresponding author e-mail: drzahoorwani@skuastkashmir.ac.in

Journal of Livestock Scienc (ISSN online 2277-6214) 12: 233-240

Received on 27/5/21, Accepted on 10/7/21, Published on 28/7/21

doi.10.33259/JLivestSci.2021.233-240

\begin{abstract}
Mitochondria and related organelles are ubiquitous among eukaryotes which may have arisen as a consequence of the endosymbiosis of an ancestral $\alpha$-proteobacterium by an ancestral eukaryote. With the passing of time, different organisms developed different types of strategies to thrive in hypoxic environments and microbial eukaryotes (protists) are no exception as these possess metabolically distinct mitochondria called mitochondrion related organelles (MROs). Among medically and veterinary important parasitic protists, there are a variety of organisms possessing MROs that reside in anaerobic niche in the body. The transformation from aerobic mitochondria to MROs occurred in independent lineages as a consequence of convergent evolution under anaerobic/microaerophilic conditions. Therefore, this review highlights the recent advances of MROs in parasitic protists.
\end{abstract}

Keywords: Aerobes; Anaerobes; Mitochondria; Mitochondrion related organelles; Protozoa 


\section{Introduction}

Protozoan infections are one among the major constraints in livestock production all over the world particularly developing countries like India (Aiman et al., 2020). Parasitic protozoa are responsible for a host of devastating diseases worldwide, including malaria, leishmaniosis, toxoplasmosis, trypanosomosis and many others. These protozoans have undergone fascinating adaptive evolutionary changes which lead to the shaping of the metabolism of these parasites. Large evolutionary distances separate free-living and parasitic protozoa from fungal, metazoan and plant lineages, concealing an intriguing evolutionary trail of gene acquisition and gene loss leading to an unusual organellar organization in their metabolism. Aerobic mitochondria with a capacity for oxidative phosphorylation are present in Plasmodium, Toxoplasma, most trypanosomatids and many other apicomplexan parasites. In the microaerophile Trichomonas vaginalis, capacity for oxidative phosphorylation is not present (Michael, 2006). There is now a prevailing consensus that all eukaryotes probably contain an organelle of mitochondrial ancestry. In response to oxygen limitation, mitochondria underwent reductive evolution as an adaptation. In some cases parasitism is influenced directly or indirectly by crucial processes compartmentalised in MROs. The electron transport system in mammals is an organized group of enzymes located in the outer membrane of the mitochondria (Ziegler et al., 1958) and a similar structure occurs in the protoplasmic membrane of bacteria (Sedar and Rudzinska, 1956). However, some protozoa also contain mitochondria resembling to those of higher animals, while others seem to have none.

In the mammalian system reduced nicotinamide nucleotides (NADPH, NADH) from the citric cycle, glycolysis and other metabolic activities are oxidized by flavoproteins, flavin adenine dinucleotide (FAD) having as their prosthetic group. Since NADP appears not to be involved in oxidative phosphorylation, the transhydrogenation takes place at the flavoprotein level where the first phosphorylation of adenosine diphosphate (ADP) occurs. Succinate is oxidized by a specific flavoprotein called succinic dehydrogenase (Sedar and Rudzinska, 1956), which is part of the citric cycle of enzymes. The flavoproteins are coupled to a complex called cytochrome reductase which includes anumber of factors such as coenzyme Q, non-heme iron, cytochrome b, cytochrome $\mathrm{c}$ and others about which considerable disagreement still exists. The experiments that have been done with protozoa have not been able to throw proper light on the problem of the composition and fine detail of this complex. This review summarizes and discusses the advances in knowledge of MROs from parasitic protists, particularly metabolic functions that contribute to parasitism and dynamics with emphasis on diversity among these organelles.

\section{Mitochondria and MROs}

Mitochondria constitute the basic electron transport complex in mammalian cells as well as parasitic protozoa where the main function of aerobic mitochondria include ATP generation via oxidative phosphorylation, heme and phospholipid synthesis, calcium homeostasis, programmed cell death, and the formation of iron- sulfur clusters (Lill and Kispal, 2000). With the help of oxidative phosphorylation and the electron transport chain (ETC), ATP generation is the major selective force for the retention of the organelle among the diverse aerobic eukaryotes. However, establishment of niches has led to various adaptations among eukaryotes. Under oxygen-restricted conditions, the mitochondria has undergone remarkable reductive modifications of its content and function by apparently combining features shared from the aerobic mitochondria with lineage-specific components and mechanisms leading to the generation of a diversified class of organelles generally called as mitochondrion-related organelles (MROs) (Santos et al., 2018). These MROs are found in a wide range of anaerobic/microaerophilic eukaryotes including several medically important parasitic protists such as Entamoeba histolytica, Giardia intestinalis, Trichomonas vaginalis, Cryptosporidium parvum, Blastocystis hominis, and Encephalitozoon cuniculi, as well as free-living protists such as Sawyeria marylandensis, Neocallimastix patriciarum, and Mastigamoeba balamuthi (Takashi and Tomoyoshi, 2014). A number of comparative studies have established that the most highly conserved function among mitochondria and MROs is Fe-S cluster biosynthesis (Lill and Kispal, 2000; Hjort et al., 2010), which was thought to be the only function that explains the almost universal existence of mitochondria and MROs in eukaryotes (Freibert et al., 2017; Ali and Nozaki, 2013). Furthermore, in the last few decades, the understanding of the role of mitochondria among the eukaryotes has expanded to that of an organelle that contributes to metabolic and cellular/organellar diversity (Rojer, 2017).

The genomic, transcriptomic, proteomic, and biochemical nature of MRO in human and animal-pathogenic protozoa has been studied owing to their medical and veterinary importance. The constituents and functions of the MROs in such anaerobic pathogenic protozoa should illuminate the evolutionary status of the MROs and also give insights into environmental constraints that drive the evolution of eukaryotes and their organelles. 


\section{Discovery of MROs}

The mitochondria originated as a consequence of endosymbiotic events commencing from the engulfment of an ancestral $\alpha$-proteobacterium by the first eukaryotic ancestor (Santos et al., 2018). Until mid of the 1990s, it was presumed that there was a eukaryote that has not gained the mitochondria (Ali and Nozaki, 2007) and the group of parasitic protists that belong to the Amoebozoa, e.g., Entamoeba histolytica, the Fornicata, e.g., Giardia intestinalis (Giardia lamblia), the Parabasalia, e.g., Trichomonas vaginalis, and the Microsporidia, were presumed to be such primitive eukaryotes which were accordingly named as the Archezoa by Cavalier-Smith (Bouzid et al., 2013).

Genes encoding Cpn60 and pyridine nucleotide transhydrogenase (PNT) found in the E. histolytica were believed to be localized in mitochondria, and phylogenetic analysis has shown that Entamoeba Cpn60 forms a monophyletic clade with Cpn60 from eukaryote mitochondria. The data was found consistent with the hypothesis that $E$. histolytica possessed mitochondria in the past and then lost them secondarily. This discovery in $E$. histolytica prompted a survey of mitochondrion-related genes in other archezoan organisms. By the late 1990s, genes encoding a 70-kD mitochondrial molecular chaperon (Hsp70), Cpn10 and Cpn60 were identified from the genome of diplomonads (G. intestinalis), parabasalids (T. vaginalis) and microsporidia (Encephalitozoon cuniculi, Nosema locustae, and Varimor phanecatrix) (Goldberg et al., 2008). Moreover, electron microscopic examination of these archezoan organisms revealed the existence of homogeneous double membrane-bound organelles containing the above-mentioned mitochondrial-type chaperones (Putignani et al., 2004; Wawrzyniak et al., 2008). These are nowadays called as mitochondrion-related organelles (MROs) generically, but the function and components are different between MROs in individual species (Tovar et al., 2003).

\section{Classification of MROs}

Mitochondria are categorized into five classes; Class 1-4 (ATP-producing) and class 5 (ATP-non producing) based on the ability to generate ATP (Muller et al., 2012). Class 1 mitochondria are "aerobic mitochondria" which exclusively use oxygen as the terminal electron acceptor. Class 2-4 mitochondria use an endogenous organic or environmental compound such as fumarate or nitrate as the electron acceptor and are referred to as anaerobic-type mitochondria. Class 5 mitochondria, are "mitosomes" and are the most reduced forms which lack ETC. they neither produce ATP nor $\mathrm{H}_{2}$ and do not use electron acceptors as well. Furthermore, class 2-4 mitochondria are classified into class 2 "anaerobic mitochondria" (which do not produce $\mathrm{H}_{2}$ but possess ETC), class 3 " $\mathrm{H}_{2}$-producing mitochondria" (which produce $\mathrm{H}_{2}$ and also possess ETC), and class 4 "hydrogenosomes" (which produce $\mathrm{H}_{2}$ and lack ETC). Mitochondrion-related organelle MROs are grouped under Class 3-5 mitochondria (Santos et al., 2018). However, the term "mitochondrion-like organelle (MLO)" is sometimes used when classification remains unassigned, e.g. MROs of Blastocystis and Cryptosporidium (Takashi and Tomoyoshi, 2014).

\section{Distribution of MROs}

In E. histolytica, G. intestinalis, T.vaginalis and Cryptosporidium spp., MROs have so far been characterized. Further researches lead to the discovery of Hydrogenosomes (class 4) as an organelle that generates ATP and hydrogen gas via fermentation as a consequence of proton reduction. Cytochromes, a membraneassociated ETC, and oxidative phosphorylation are lacking in Hydrogenosomes. They occur in trichomonads, the ciliates, amoeba and some fungi. Mitosomes (class 5) on the other hand, produce, neither hydrogen gas nor ATP, and are found in Giardia, Microsporidia, Cryptosporidium and Entamoeba. In Blastocystis, which is a common inhabitant of the human gastrointestinal tract and a strict anaerobe, its MRO is referred as MLO, but should be refined as the $\mathrm{H}_{2}$-producing mitochondria, as it retains respiratory complex I and an organelle genome, and its components and functions are more similar to hydrogenosomes than to mitosomes (class 3) (Takashi and Tomoyoshi, 2014).

\section{Morphological features of MROs}

The mitochondria are thought to be derived via endosymbiosis, and as demonstrated by electron microscopy these along with MROs are surrounded by double membranes of lipid bilayer. Aerobic mitochondria possess a genome (Susin et al., 1999), MROs, with a few exceptions contain no genome (Goldberg et al., 2008). The lack of organelle genome was demonstrated in most cases by lack of SYTO-61 and DAPI staining of extranuclear DNA or in situ nick-translation coupled to immunofluorescence microscopy. All MROs from parasitic protists, except $B$. hominis do not appear to retain cristae, which develop well in the mitochondria from aerobic organisms to expand the surface area of the inner mitochondrial membrane, enhancing its ability to produce ATP. Mitochondria 
from parasites like Cryptosporidium spp. utilize oxygen as a terminal electron acceptor, and thus they are probably classified into class 1 or 2.

\section{Tricarboxylic acid cycle, electron transport chain and energy production}

MROs have lost majority of proteins involved in energy generation via the tricarboxylic acid cycle, oxidative phosphorylation, and beta oxidation of fatty acids. However, in parasitic protists (classes 3 and 4), some anaerobic MROs can still produce ATP via a part of components for respiratory chain (complex I) or substrate-level phosphorylation (e.g., class 4, Trichomonas) (Takashi and Tomoyoshi, 2014).

\section{Hydrogenosomes in Trichomonas spp.}

.Hydrogenosomes are found in multiple lineages in Trichomonas. The best knowledge of energy production in hydrogenosomes (class 4) is described on the basis of Trichomonos. Oxygen uptake in Trichomonas foetus, $T$. vaginalis, and $T$. gallinae is probably mediated by flavoproteins (Wawrzyniak et al., 2008). All three organisms depend on conventional glycolysis supplemented with other systems linked to NADP and succinate to furnish electron donors. T. vaginalis hydrogenosomes contain malic enzyme (ME), [Fe]-hydrogenase, ferredoxin, pyruvate: ferredoxin oxidoreductase (PFOR), adenylate kinase, acetate: succinate CoA transferase (ASCT), succinate thokinase a and b subunits, NADH dehydrogenase 24- and 51-kDa subunits, and oxygen scavenging system including thioredoxin (Trx), Trx peroxidase, rubrerythrin, iron superoxide dismutase. These enzymes carry out the substrate level phosphorylation and fermentation to generate equimolar of ATP, acetate, $\mathrm{CO}_{2}$, and $\mathrm{H}_{2}$ from pyruvate or malate. Pyruvate is decarboxylated by PFOR, generating $\mathrm{CO}_{2}$, Acetyl-CoA, and reduced ferredoxin, the latter of which in turn carries electrons to ferredoxin-dependent $[\mathrm{Fe}]$-hydrogenase. [Fe]-hydrogenase further donates electrons to protons to generate molecular hydrogen. The CoA moiety of acetyl-CoA is transferred to succinate by ASCT, to form succinyl-CoA and acetate as an end product. Succinyl-CoA synthetase finally yields ATP through substrate-level phosphorylation (Tovar et al., 2003; Goldberg et al., 2008).

\section{Mitochondria and MROs of Cryptosporidium}

Components and pathways to generate ATP in mitochondria or MROs seem to be different in different Cryptosporidium species (Mogi and Kita, 2010). C. parvum and C. hominis, infect human intestine and their mitosomes lack pyruvate dehydrogenase, most of the TCA cycle enzymes except malate-quinoneoxidoreductase, and most of subunits of ATP synthase except $\mathrm{a}$ and $\mathrm{b}$ subunits. Hence, in these two Cryptosporidium species, ATP appears to be generated only by substrate-level phosphorylation. However, in Cryptosporidium muris, a genomewide survey has shown that all TCA cycle enzymes and ATP synthase are conserved. Therefore, C. muris is likely capable of oxidative phosphorylation with pyruvate- NADP oxidoreductase and a simple and unique respiratory chain consisting of pyridine nucleotide transhydrogenase, alternative NADH dehydrogenase and cyanide-insensitive alternative oxidase (AOX), the latter passes an electron from ubiquinol to oxygen to yield $\mathrm{H}_{2} \mathrm{O}$ (Tovar et al., 1999).

\section{MROs of Giardia}

Mitosomes in G. intestinalis are not directly involved in energy metabolism (class 5), but in G. intestinalis, like E. histolytica, PFOR and [Fe]-hydrogenase enzymes are in the cytosol. Although G. intestinalis produces molecular hydrogen under strictly anoxic conditions (Lloyd et al., 2002), ATP is not generated in the mitosomes (Muller et al., 2012). However, it was previously demonstrated by proteome analysis that a novel diflavo-protein is isolated from the mitosomes. The diflavo-protein, named GiOR-1, has the mitosomal ferredoxin-independent and NADPH-dependent reductase activity, suggesting that the electron transport by pyridine nucleotides exists in Giardia mitosomes (Jedelsky et al., 2011).

\section{MROs of Amoebozoa}

The mitosomes in Entamoeba histolytica are the best-studied MROs in the super group Amoebozoa (Mi-ichi et al., 2009; Dolezal et al., 2010). Similar to Giardia, the extended glycolysis and ACS-catalysed ATP synthesis pathway of Entamoeba is localized exclusively in the cytoplasm. However, the ISC system which is involved in the biosynthesis of $\mathrm{Fe}-\mathrm{S}$ clusters is lost in this organism, and got replaced with a homologous system called the nitrogen fixation (NIF) system which was acquired by LGT from e-proteobacteria (Stairs et al., 2015). Earlier reports suggested that the NIF system is dually localized in the cytoplasm (Maralikova et al., 2010), but more recent proteomic studies were unable to detect NIF system components in the organelle. Therefore the notion that the main reason for maintaining MROs is the biosynthesis of Fe-S clusters still remains unanswered (Stairs et al., 2015). 
Today the known function of the MROs in Entamoeba is ATP import via mitochondrial carrier proteins (Stairs et al., 2015) and sulfate activation for the generation of sulfated compounds (Mi-ichi et al., 2009; Miichi et al., 2015).

\section{General electron transport in few other protozoa}

Among the parasitic protozoa only a few species have been studied in the detail necessary for characterizing the various components of the electron transport chain.

\section{Plasmodium}

Our biochemical information of the Plasmodia is limited to the erythrocytic stage in the life cycle of the organism. Several species have been studied, but since no significant differences have been discovered, so to treat them as a group seems best. No attempts to prepare pure enzymes has yet been made. Early work utilized infected blood of monkeys with uninfected blood as control. Further, washed parasitized avian erythrocytes were used. Saponin and specific hemolysins have been employed to prepare free parasites, and finally homogenates of free parasites have been studied for individual enzyme activities (Baernstein, 1963) With these preparations, several reactions of phosphorylative glycolysis, including the oxidation of 3-phosphoglyceraldehyde by NAD has been demonstrated. Lactic acid accumulates anaerobically, and to a lesser extent aerobically. NADH produced in the first reaction is reoxidized by pyruvate in the second and another coupling was demonstrated by showing lactate oxidation by indophenols requiring flavoprotein, which may be part of the electron transport chain. The utilization of glycerol by $P$. knowlesi probably indicates the presence of another system for the reduction of NAD and oxidation of NADH, by glycerophosphate, although no evidence for phosphorylation was found. Recent studies have found adequate evidence for phosphorylation as well as for a functional citric cycle and free parasites utilized most of the cycle components. Pyruvate oxidation was stimulated by dicarboxylic acids and completely inhibited by malonate. Fumarate or malate relieved the inhibition on succinic dehydrogenase. So, the electron transport chain in Plasmodia contains proteins for reduction of NAD and perhaps NADP. Malonate inhibited oxygen uptake with succinate as substrate and is reversed by fumarate and malate (Baernstein, 1963). This indicates flavoprotein activity. The isolation of FAD from parasites, as well as reduction of indophenol and cresyl blue, support this interpretation. The oxidases being cyanide sensitive, among which one is azide sensitive, and one is inhibited by carbon monoxide. Though no supporting evidence has been obtained, but it indicates that these oxidases may be related to cytochrome oxidase. The only evidence for intermediate carriers so far found is the inhibition of oxygen uptake by naphthoquinones which in other organisms act upon cytochrome reductase (Baernstein, 1963; Takashi and Tomoyoshi, 2014).

\section{Trypanosomes}

Trypanosoma spp., a veterinary protozoan serves as a model for Trypanosoma rhodesiense and Trypanosoma gambiense, which cause human diseases known as African sleeping sickness. The bloodstream form of this parasite has neither oxidative phosphorylation, nor cytochrome-mediated electron transport system, as well as no tricarboxylic acid cycle. It has been seen that the high rate of oxygen consumption by these protozoa is not inhibited by cyanide, antimycin A, rotenone or azide and these parasites are totally dependent on glycolysis for their energy production. The respiratory system has been observed to be composed of glycerol-3-phosphate dehydrogenase and an oxidase. The respiratory system is linked to glycerol 3-phosphate shuttle. The shuttle and the respiratory system reoxidize NADH to NAD+ thus allowing continuous glycolysis. In the trypanosome most steps of glycolysis take place in a specialized microbody called a glycosome (Sutak et al., 2004; Goldberg et al., 2008). Though, the electron transport system has not been well understood, but the respiratory system re-oxidizes the sn-glycerol3-phosphate produced by NADH-mediated reduction of dihydroxyacetone phosphate in the glycosome (Takashi and Tomoyoshi, 2014).

The blood stream forms of vivax, congolense and brucei groups are characterized by high rates of glycolysis, low activity of the citric cycle enzymes, and active oxidases which are not inhibited by cyanide, azide, or carbon monoxide. Glycerophosphate plays a unique role as substrate for two enzymes, one linked to NAD and the other probably linked to FAD. The absence of hydrogen peroxide as end product has not been explained. It cannot be referred to the reaction with pyruvate as it would result in elimination of large amounts of carbon dioxide and acetate, none of them are found in cultures of the organisms. The absence of peroxide can neither be attributed to the action of catalase or peroxidase since these enzymes are not present in the organisms (Takashi and Tomoyoshi, 2014). The sensitivity of these terminal oxidases to sulfhydryl reagents indicates that perhaps another factor coupling flavoprotein to oxygen may be present. 
Biochemical studies of trypanosomes are much more extensive than those of any other species of parasitic protozoan. The organisms are somewhat easier to obtain than are Plasmodia or Amoebae, although forms inhabiting the blood stream are sometimes difficult to separate from white blood cells and this has led to confusion regarding the presence of cyanide sensitive oxidases (Ali and Nozaki, 2007; Takashi and Tomoyoshi, 2014). The oxidation of glycerophosphate has recently assumed considerable importance as the terminal respiratory enzyme reaction of the trypanosomes lacking the cytochrome system. Several evidences indicate the presence of the citric cycle in those trypanosomes which are sensitive to cyanide.

\section{Protozoan mitochondria as potent therapeutic target and a possible vaccine candidate}

Chemotherapy is the primary means of treating any parasitic infections. A problem for chemotherapy is to find a novel and potential molecule in parasites particularly protozoa, which could be exploited as drug target. To acheive this goal, mitochondrion of protozoa can be considered as the most valuable and potential target organelle because of its unique structure and function compared to their natural host. In fact, the respiratory systems of parasitic protozoa typically show greater diversity in electron pathways than do their mammalian hosts. These unique aspects of electron transport chain (ETC) complexes and their related enzymes represent promising targets for chemotherapy. A cytochrome independent Alternative Oxidase (AOX) in parasites is a leading drug target. Topoisomerases play key functions in replication and organization of kDNA, present in kinetoplast. They are also considered as potential targets for anti-parasitic drugs. Moreover, a novel pathway of type II Fatty acid synthesis in mitochondria of trypanosomatids provides a new array of inhibitors that could be effective against these parasites (Sen and Majumder, 2008). Also, programmed cell death in the unicellular organism has attracted considerable attention, because several studies have shown that different drugs like pentamidine, miltefosine, and topoisomerase induce parasites to die via apoptotic cell death through the mitochondrion-dependent pathway against parasites like Leishmania, Trypanosoma, Plasmodium, and Toxoplasma (Menna-Barreto, 2008). Recent studies on the emergence of drug resistance severely limit the arsenal of available drugs against protozoan parasites. Particularly, mutations of cytochrome $b$ gene of ETC or changes in iron homeostasis by mitochondrial enzyme aconitase alter sensitivity of MDR1 and regulate resistance level to anti-parasitic drugs (Sen and Majumder, 2008). Thus the study on action of therapeutically applied substances on mitochondria can have potential application in the future.

Drugs remain central to alleviating clinical disease and for larger scale disease control programmes. However, available drugs being in use for decades have resulted in drug resistance which is threatening the sustainable parasite control. These facts have diverted the scientists to make efforts on vaccine development. But, there are a number of factors which hinders the development of a successful protozoan vaccine like their complex life cycle, multiple developmental stages and sub clinical form of disease. With the recent advancement in immunology, a continuous flow of vaccines to the market is going on, but among them the percentage of parasitic vaccine is very low (Vercruysse et al., 2004). One of the most important protozoa of is the Trypanasoma spp. which causes a serious problem in case of animals as well as humans (La Greca and Magez, 2011). But an important feature lying with this protozoa is that it can change its antigenic surface protein in such a rapid manner that the immune system of host cannot trace it (Pays et al., 2004). In Certain protozoa, sometimes a single host cell is infected with protozoa at different stages of development giving a feel to the host immune system that there a number of protozoa in a single cell (Vercruysse et al., 2007) and host immune system is not able to make antibodies against a particular life cycle stage. However, efforts for making economically feasible and effective protozoan vaccine, are still in continuation and mitochondrion related organelles can act as one among such candidates as the enzymes involved in MRO can be targeted for vaccine candidates, but we are still far from that research prospectus.

\section{Conclusive remarks}

The reductive evolution from aerobic mitochondria to MROs apparently seems convergent; though, differences in components, functions, and morphological features among MROs exist (Takashi and Tomoyoshi, 2014), which may have been generated by a secondary gene loss and/or lateral gene transfer (Rojer et al., 2017; Leger et al., 2017). The number of organisms possessing MRO is expected to increase by increased genome sequencing of anaerobic/microaerophilic unicellular eukaryotes. Thus the diversity of MROs and their function will increase accordingly. A highly sensitive proteomic analysis of highly purified MROs by techniques like conventional physical separation, immunoprecipitation, and FACS-based separation of organelles is also the need of hour to demonstrate the components involved in the biogenesis of MROs so to provide insight into the evolutionary constraints that drive organelle diversification.

MROs can be targeted for the development of chemotherapeutics against infections caused by MRO-possessing pathogens. E.g., the sulfate activation pathway in mitosomes of E. histolytica can be exploited as a drug target if 
delivering route to mitosomes becomes established. At present time, complete or partial genome sequences are now available for several medically and evolutionarily important parasitic protozoa and by the application of bioinformatics, complete metabolic stock of these parasites can be predicted. The establishment of genome-editing techniques such as the CRISPR/ Cas9 system, which has been accomplished in Cryptosporidium (Vinayak et al., 2015) and Trichomonas (Janssen et al., 2018), would be beneficial in not only understanding MROs and its evolution, but also about parasitism in general.

\section{References}

1) Aiman, A., Shahardar, R.A., Bulbul, K.H., Wani, Z.A., Allaie, I.M., Makhdoomi, D. M., Hina, F.B. and Rather, M.A. 2020. Anticoccidial efficacy of Curcuma longa (turmeric) and Zingiber officinale (ginger) in goats in central Kashmir. Journal of Pharmacognosy and Phytochemistry 9: 354-360.

2) Ali, V. and Nozaki, T. 2007. Current therapeutics, their problems, and sulfur-containingamino-acid metabolism as a novel target against infections by "amitochondriate" protozoan parasites. Clinical Microbiology Reviews 20: 164-187.

3) Ali, V. and Nozaki, T. 2013. Iron-sulphur clusters, their biosynthesis and biological functions in protozoan parasites. Advances in Parasitology 83: 1-92

4) Baernstein, H.D. 1963. Electron Transport Mechanisms in Parasitic Protozoa. The Journal of Parasitology 49: 12-21.

5) Bouzid, M., Hunter, P.R., Chalmers, R.M. and Tyler, K.M. 2013. Cryptosporidium pathogenicity and virulence. Clinical Microbiology Reviews 26: 115-134.

6) Dolezal, P., Michael. J.D., Maya. K., Peter, W., Vladimir, A. L., Jung, H. F. et al. 2010. The essentials of protein import in the degenerate mitochondrion of Entamoeba histolytica. PLOS Pathogens 6: e1000812. (doi:10.1371/journal.ppat.1000812).

7) Freibert, S.A., Alina, V.G., Christian, H., Sabine, M., Paul, D., Tom, A.W., Sirintra, N., Shaojun, L., Kacper, S., Eckhard, B., Eva, H., Robert, P.H., John, M.L.T., Martin, E. Roland, L. 2017. Evolutionary conservation and in vitro reconstitution of microsporidian iron-sulfur cluster biosynthesis. Nature Communications 8: 1-12.

8) Goldberg, A.V., Molik, S., Tsaousis, A.D., Neumann, K., Kuhnke, G., Delbac, F., Vivares, C.P., Hirt, R. P., Lill. R. and Embley, T.M. 2008. Localization and functionality of microsporidian iron-sulphur cluster assembly proteins. Nature 452: 624-628.

9) Hjort, K., Alina, V.G., Anastasios, D.T., Robert, P.H. and Embley, T.M. 2010. Diversity and reductive evolution of mitochondriaamong microbial eukaryotes. Philosophical Transactions of Royal Society B Biological Sciences 365: 713-727.

10) Janssen, B.D., Yi-Pei, C., Brenda, M.M., Shuqi, E.W., Augusto, S. and Patricia, J.J. 2018. CRISPR/Cas9mediated gene modification and gene knock out in the human-infective parasite Trichomonas vaginalis. Scientific Reports 8: 1-14

11) Jedelský, P.L., Dolezal, P., Rada, P., Pyrih, J., Smíd, O., Hrdý, I., Sedinová, M., Marcinciková, M., Voleman, L., Perry, A.J., Beltrán, N.C., Lithgow, T. and Tachezy, J. 2011. The minimal proteome in the reduced mitochondrion of the parasitic protest Giardia intestinalis, PLoS One 6 e17285.

12) La Greca, F. and Magez, S. 2011. Vaccination against trypanosomiasis: Can it be done or is the trypanosome truly the ultimate immune destroyer and escape artist? Human Vaccines 7: 1225-1233.

13) Leger, M.M., Martin, K., Ryoma, K., Courtney, W.S., Keitaro, K., Ivan, C., Jeffrey, D. S., Jan, O.A., Feifei, Xu., Akinori, Y., Laura, E., Qianqian, Z., Kiyotaka, T., Yuji, I., Alastair, G.B.S., Tetsuo, H. and Andrew J.R. 2017. Organelles that illuminate the origins of Trichomonas hydrogenosomes and Giardia mitosomes. Nature Ecology and Evolution 1: 92.

14) Lill, R. and Kispal, G. 2000. Maturation of cellular Fe-S proteins: an essential function of mitochondria. Trends in Biochemical Sciences 25: 352-356.

15) Lloyd, D., Ralphs, J.R. and Harris, J.C. 2002. Giardia intestinalis, a eukaryote without hydrogenosomes, produces hydrogen. Microbiology 148: 727-733.

16) Maralikova, B., Ali, V., Nakada-Tsukui, K., Nozaki, T., van der Giezen, M., Henze, K. and Tovar, J. 2010. Bacterial-type oxygen detoxification and iron-sulfur cluster assembly in amoebal relict mitochondria. Cellular Microbiology 12: 331-342.

17) Menna-Barreto, R.F. 2008. Different cell death pathways induced by drugs in Trypanosoma cruzi: an ultrastructural study. Micron 40: 157-168 
18) Michael L.G. 2006. Niche metabolism in parasitic protozoa.Philosophical Transactions of the Royal Society B 361: 101-118.

19) Mi-ichi, F., Abu Yousuf, M., Nakada-Tsukui, K. and Nozaki, T. 2009. Mitosomes in Entamoeba histolytica contain a sulfate activation pathway. Proceedings of the National Academy of Sciences of the USA 106: 731736. (doi:10.1073/pnas. 0907106106).

20) Mi-ichi, F., Miyamoto, T., Takao, S., Jeelani, G., Hashimoto, T., Hara, H., Nozaki, T. and Yoshida, H. 2015. Entamoeba mitosomes play an important role in encystation by association with cholesteryl sulfate synthesis. Proceedings of the National Academy of Sciences of the USA 112.

21) Mogi, T. and Kita, K. 2010. Diversity in mitochondrial metabolic pathways in parasitic protists Plasmodium and Cryptosporidium. Parasitology International 59: 305-312.

22) Muller, M., Mentel, M., Hellemond, J.J., Henze, K., Woehle, C., Gould, S.B., Re-Young, Y., Giezen, M., Tielens, A.G.M. and William, F.M. 2012. Biochemistry and evolution of anaerobic energy metabolism in eukaryotes. Microbiology and Molecular Biology Reviews 76: 444-495.

23) Pays, E., Vanhamme, L. and Perez-Morga, D. 2004. Antigenic variation in Trypanosoma brucei: Facts, challenges and mysteries. Current Opinion Microbiology 7: 369-374.

24) Putignani, L., Tait, A., Smith, H.V., Horner, D., Tovar, J.L., Tetley, M. and Wastling, J. M. 2004. Characterization of a mitochondrion-like organelle in Cryptosporidium parvum. Parasitology 129: 1-18.

25) Roger, A.J., Muñoz-Gómez, S.A. and Kamikawa, R. 2017. The origin and diversification of mitochondria. Current Biology 27: 1177-1192.

26) Santos, H. J., Makiuchi, T. and Nozaki, T. 2018. Reinventing an Organelle: The Reduced Mitochondrion in Parasitic Protists. Trends in Parasitology 20: 1-18.

27) Sedar, A.W. and M.A. Rudzinska. 1956. Mitochondria of protozoa. Journal of Biophysical and Biochemical Cytology 4: 331-334.

28) Sen, N. and Majumder, H.K. 2008. Mitochondrion of protozoan parasite emerges as potent therapeutic target: Exciting drugs are on the horizon. Current Pharmaceutical Design 14: 839-846

29) Stairs, C.W., Leger, M.M. and Roger, A.J. 2015. Diversity and origins of anaerobic metabolism in mitochondria and related organelles. Philosophical Transactions of the Royal Society B 370: 1-13.

30) Susin, S.A., Lorenzo, H.K., Zamzami, N., Marzo, I., Snow, B.E., Brothers, G.M., Mangion, J., Jacotot, E., Costantini, P. Loeffler, M., Larochette, N., Goodlett, D.R., Aebersold, R., Siderovski, D.P., Penninger, J.M. and Kroemer, G. 1999. Molecular characterization of mitochondrial apoptosis-inducing factor. Nature 397: 441-446.

31) Sutak, R., Dolezal, P., Fiumera, H.L., Hrdy, I., Dancis, A., Delgadillo-Correa, M., .Johnson, P.J., Müller, M. and Tachezy, J. 2004. Mitochondrial-type assembly of FeS centers in the hydrogenosomes of the amitochondriate eukaryote Trichomonas vaginalis, Proceedings of National Academy of Sciences of the USA 101: 1036810373.

32) Takashi, M. and Tokomoshi, N. 2014. Highly divergent mitochondrion-related organelles in anaerobic parasitic protozoa Biochimie 3-17

33) Tovar, J., Fischer, A. and Clark, C.G. 1999. The mitosome, a novel organelle related to mitochondria in the amitochondrial parasite Entamoeba histolytica. Molecular Microbiology 32: 1013-1021.

34) Tovar, J., León-Avila, G., Sánchez, L.B., Sutak, R., Tachezy, J., van der Giezen, M., Hernández, M., Müller, M. and Lucocq, J. M. 2003. Mitochondrial remnant organelles of Giardia function in iron-sulphur protein maturation. Nature 426: 172-176.

35) Vercruysse, J., Knox, D.P., Schetters, T.P. and Willadsen, P. 2004. Veterinary parasitic vaccines: Pitfalls and future directions. Trends in Parasitology 20: 488-492.

36) Vercruysse, J., Schetters, T.P., Knox, D.P., Willadsen, P. and E. Claerebout. 2007. Control of parasitic disease using vaccines: An answer to drug resistance. Revue Scientifique et Technique 26: 105-115.

37) Vinayak, S., Pawlowic, M.C., Sateriale, A., Brooks, C.F., Studstill, C.J., Bar-Peled, Y., Cipriano, M.J. and Striepen, B. 2015. Genetic modification of the diarrhoeal pathogen Cryptosporidium parvum. Nature 523: 477480

38) Wawrzyniak, I., Roussel, M., Diogon, M., Couloux, A., Texier, C., Tan, K.S., Vivarès, C.P., .Delbac, F., Wincker, P. and Alaoui, H.E. 2008. Complete circular DNA in the mitochondria-like organelles of Blastocystis hominis. International Journal of Parasitology 38: 1377-1382.

39) Ziegler, D.M., Linnane, A.W., Green, D.E., Dass, C.M.S. and H. Ris. 1958. Studies on the electron transport system. XI. Correlation of the morphology and enzymatic properties of mitochondrial and sub-mitochondrial particles. Biochimica and Biophysica Acta 28: 524-538. 